\title{
Chronic Mercury Intoxication Masquerading as Systemic Disease: A Case Report and Review of the Literature
}

\author{
Alexandre Malek ${ }^{1,2}$, Krystel Aouad ${ }^{3}$, Rana El Khoury ${ }^{4}$, Maya Halabi-Tawil ${ }^{4}$, Jacques Choucair ${ }^{1}$ \\ ${ }^{1}$ Infectious Diseases Department, Saint Joseph University, Faculty of Medicine/Hotel Dieu De France Hospital, Beyrouth, Lebanon \\ 2Université Pierre et Marie Curie, Paris, France \\ ${ }^{3}$ Internal Medicine, Saint Joseph University, Faculty of Medicine/Hotel Dieu De France Hospital, Beyrouth, Lebanon \\ ${ }^{4}$ Dermatology Department, Saint Joseph University, Faculty of Medicine/Hotel Dieu De France Hospital, Beyrouth, Lebanon
}

\section{Doi: 10.12890/2017_000623 - European Journal of Case Reports in Internal Medicine - @ EFIM 2017}

Received: $17 / 04 / 2017$

Accepted: 17/05/2017

Published: 24/05/2017

How to cite this article: Malek A, Aouad K, El Khoury R, Halabi-Tawil N, Choucair J. Chronic mercury intoxication masquerading as systemic disease: a case report and review of the literature. EJCRIM 2017;4: doi:10.12890/2017_000623.

Conflicts of Interests: The Authors declare that there are no competing interests.

Acknowledgements: A. Malek, K. Aouad and J. Choucair contributed equally to this work. R. El Khoury and M. Halabi-Tawil contributed equally to this work. The Authors would like to thank the patient for his participation.

This article is licensed under a Commons Attribution Non-Commercial 4.0 License

\section{ABSTRACT}

Background: Mercury is a highly toxic environmental metal that exists in three different forms: elemental, inorganic and organic. Intoxication occurs in either occupational or non-occupational settings, mainly after the inhalation of vapour and fumes in work places, laboratories or homes. Chronic mercury toxicity ranges from mild and insignificant to severe and life-threatening. We describe the case of a young male patient who presented with multiple organ dysfunction after chronic mercury exposure.

Case presentation: We report the case of 28-year-old male artisanal gold miner who was admitted to hospital for severe neurological impairment associated with inflammatory bowel disease-like symptoms and a skin rash after mercury exposure. Symptomatic treatment and corticosteroid administration assured rapid clinical improvement. Chronic mercury poisoning can masquerade as an autoimmune or systemic inflammatory disease.

Conclusion: Physicians should be aware that low exposure to mercury, even from artisanal gold mining, may be harmful to health. Management can be simple without the need for aggressive or invasive therapeutic measures. Larger case series are required in order to establish a clear management plan.

\section{LEARNING POINTS}

- Mercury intoxication has a wide the variety of clinical manifestations that may involve the neurological, gastrointestinal and dermatological systems.

- Therefore, it can mimic degenerative neurological conditions, autoimmune diseases, as well as metabolic and mitochondrial disorders.

- Once diagnosed, mercury intoxication is easily treated.

\section{KEYWORDS}

Mercury; chronic Intoxication; heavy metal; inflammatory disease 


\section{INTRODUCTION}

Mercury is a highly toxic environmental metal that exists in three different forms: elemental, inorganic (mercuric salts, e.g. mercuric chloride) and organic (e.g. methylmercury) ${ }^{[1,2]}$. It exhibits several patterns of toxicity depending on the chemical form and route of exposure. Intoxication occurs in either occupational or non-occupational settings, mainly after inhalation of vapour and fumes in work places, laboratories or homes ${ }^{[2]}$. Inorganic mercury can only be absorbed by the gastrointestinal tract and skin, while organic mercury can be absorbed by the gastrointestinal tract, skin and respiratory system ${ }^{[1-3]}$.

Chronic mercury toxicity ranges from mild and insignificant to severe and life-threatening, inducing a wide array of clinical manifestations. The central nervous system, gastrointestinal tract, kidneys, skin and mucosae can be affected ${ }^{[3]}$.

We report the case of a young male patient who was admitted to hospital for severe neurological impairment associated with inflammatory bowel disease-like symptoms and a skin rash after mercury exposure.

\section{CASE DESCRIPTION}

A 28-year-old male was admitted to our hospital with a 3-month history of low-grade fever, severe insomnia, behavioural changes, visual hallucinations, recent hearing difficulty and unintentional $12 \mathrm{~kg}$ weight loss. His past medical history was notable for slowly progressive ataxia along with lower limb paraesthesia, burning sensations and muscular fasciculations, followed by the sensation of incomplete bladder voiding, erectile dysfunction and urinary retention. He had also developed a pruritic cutaneous rash with palmoplantar scaling and severe spasmodic abdominal pain accompanied by bloody diarrhoea. Previous investigations had excluded infectious, metabolic, neurological and mitochondrial pathologies. The patient had started working 6 months previously in Liberia at an artisanal gold mine with exposure to mercury-gold amalgam. He did not report any contact allergies, at-risk sexual activities or relevant family history.

On physical examination, the patient was stable. His blood pressure was $110 / 65 \mathrm{mmHg}$, body temperature $38^{\circ} \mathrm{C}$, pulse rate 102 beats/ minute and respiratory rate 20 breaths/minute. He was conscious, cooperant and oriented but easily became agitated and irritable. Heart beats were regular, lungs were clear, and the abdomen was tender in the right lower quadrant without any other abnormalities. Neurological examination showed a severe ataxic gait, an absence of deep tendon reflexes, and diffuse muscle fasciculations mainly in the lower limbs. He had no motor weakness or sensory disturbance. The rest of the neurological examination was normal. Skin examination showed an erythematous macular rash with multiple excoriations on the trunk and some pustules on the back and chest, along with excoriated papules and pustules on the back, axillae and periumbilical area (Fig. 1). We noted a clearly demarcated incision on the patient's back that had been made to allow bloodletting, which in traditional African medicine is believed to help eliminate toxins from the blood. In addition, palmoplantar desquamation was prominent (Fig. 2). No other pathological findings were observed on physical examination, ruling out lymphadenopathies, arthritis and testis anomalies.

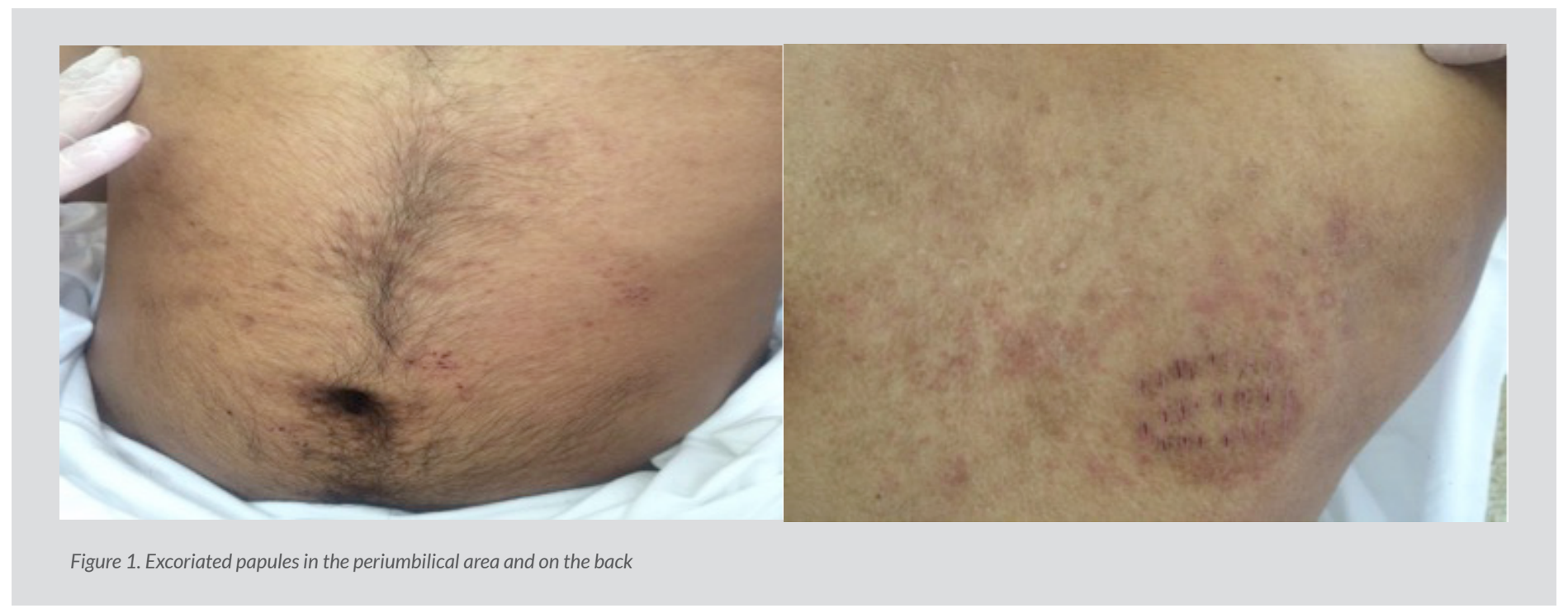

Laboratory tests results showed mild leukocytosis (neutrophils 72\%) and normal haemoglobin and platelet levels, erythrocyte sedimentation rate, C-reactive protein, liver function tests, lactate dehydrogenase, kidney function, and biochemistry profile except for mild hypokalaemia (3.1 mEq/l). Urine analysis showed 80 white blood cells (WBC), 70 red blood cells (RBC), with sterile culture and proteinuria of $1.125 \mathrm{~g} / \mathrm{day}$. 


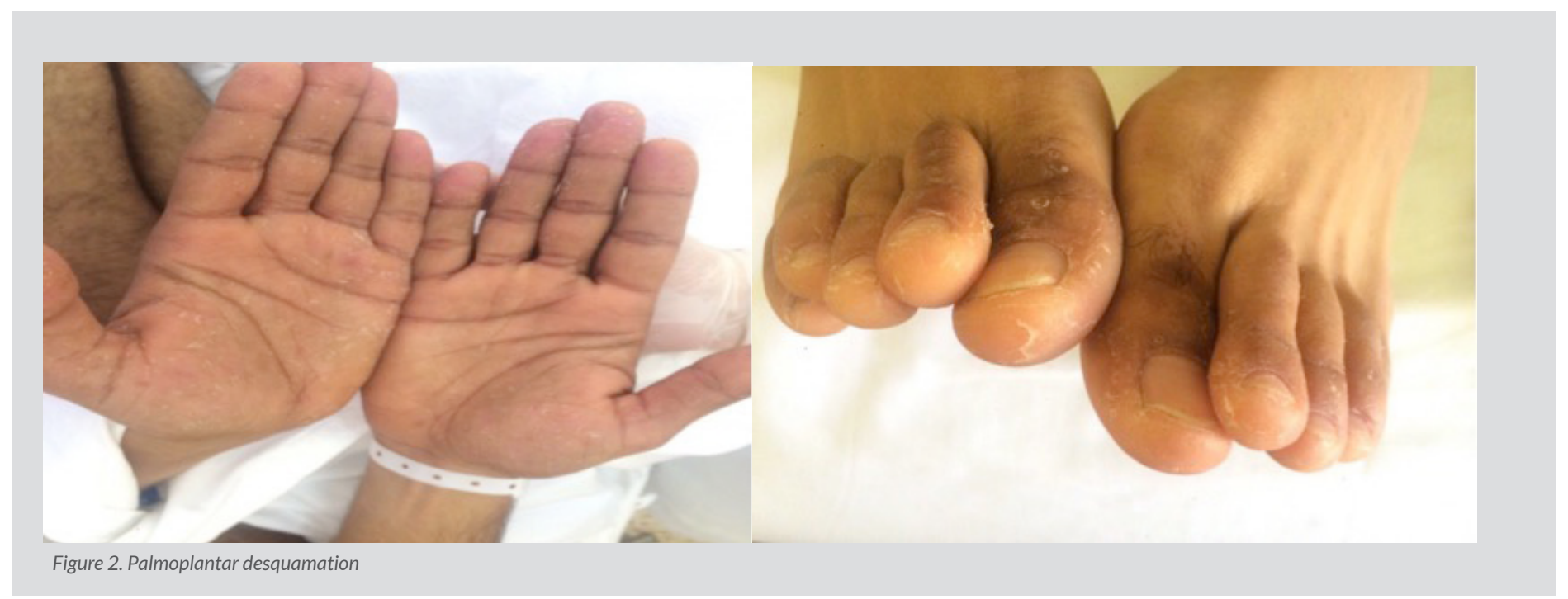

An extensive serology workup showed negative Weil-Felix, Wright, Widal and venereal disease research laboratory (VDRL) tests, a negative Treponema pallidum hemagglutination assay (TPHA), and negative tests for hepatitis B virus (HBV), hepatitis C virus (HCV), human immunodeficiency virus (HIV) 1 and 2, and malaria. The immunological profile showed normal levels of antinuclear antibodies (ANA), C3, $\mathrm{C} 4$, anti-thyroid-stimulating hormone (TSH) receptor antibodies and normal serum immunoelectrophoresis. A purified protein derivative (PPD) skin test was negative. Tests for vitamin B12, TSH and ceruloplasmin were all within normal limits. Culture of skin pustule content was sterile. A lumbar puncture showed no abnormalities.

An electrocardiogram (EKG) showed sinus tachycardia. Chest x-ray was normal. Cerebral, pituitary and dorso-lumbar magnetic resonance imaging (MRI) was normal. Abdominal and pelvic ultrasound revealed chronic cystitis and a thickened bladder wall. Electromyography showed generalized muscular fasciculations due to toxic, metabolic or endocrine causes. A neuromuscular biopsy was unremarkable, while a skin biopsy specimen from a pustule on the back showed non-specific mild chronic dermatitis. Colonoscopy demonstrated mild ileitis and histopathology disclosed granulomatous lesions suggestive of Crohn's disease or other mimicking diseases.

In accordance with the patient's clinical picture and risk of severe occupational exposure to heavy metals, plasma levels showed high concentrations of mercury at $8.83 \mu \mathrm{g} / \mathrm{l}$ or $44 \mathrm{nmol} / \mathrm{I}$ (normal level $<5 \mu \mathrm{g} / \mathrm{l}$ or $<25 \mathrm{nmol} / \mathrm{l}$ ), chrome at $1.23 \mu \mathrm{g} / \mathrm{l}$ (normal level $<0.87 \mu \mathrm{g} / \mathrm{l}$ ) and nickel at $2.5 \mu \mathrm{g} / \mathrm{l}$ (normal level $<1.3 \mu \mathrm{g} / \mathrm{l}$ ), while other metals were within the normal range.

A diagnosis of heavy metal intoxication was made. The patient was started on a high-dose corticosteroid (80 mg methylprednisolone per day) for 2 weeks followed by dose tapering over 2 months. His hospital course was uneventful with a smooth recovery. The patient was discharged with regular follow-up in the outpatient clinic. A remarkable improvement in the ataxia and other neurological complaints and gastrointestinal symptoms was noted at follow-up 2 months after corticosteroid cessation. One year later, the patient is asymptomatic and did not resume his work as an artisanal gold miner.

\section{DISCUSSION}

Heavy metal intoxication developed as a major healthcare problem especially after the industrial revolution. Elemental mercury is still widely used in industrial facilities, households, and in medical and electrical devices (thermometers, thermostats, electrical switches, dental amalgam $)^{[4]}$. It is also used in artisanal small-scale gold mining ${ }^{[4]}$ where gold extraction using archaic and unsafe methods predisposes miners to mercury poisoning mainly through inhalation as in our patient.

Mercury is a toxin that eventually affects cellular physiology and haemostasis. Toxicity varies depending on the form of mercury, dose, rate of exposure and the pharmacokinetic characteristics of each form ${ }^{[1,3,5]}$. Mercury vapour inhalation mainly affects the brain, while inorganic mercury and methylmercury primarily affect the intestinal mucosae and kidneys ${ }^{[1]}$. Acute exposure to elemental mercury vapour can cause severe pneumonitis ${ }^{[1]}$. Clinical findings are systemic and more subtle in chronic exposure.

Mercury poisoning poses a diagnostic challenge. Firstly, the blood toxicity threshold has been debated. Graeme et al. considered that a blood level above $15 \mathrm{~g} / \mathrm{l}$ indicated mercury toxicity ${ }^{[6]}$, while Dantzig found that a level as low as $6 \mathrm{~g} / \mathrm{l}$ or less may be toxic ${ }^{[7]}$. Hence, symptoms may be present at low levels of exposure ${ }^{[8,9]}$. Secondly, there are no defined criteria for the diagnosis of mercury poisoning since there is no clear correlation between clinical symptoms of intoxication and the level of mercury in the body ${ }^{[3,8,9]}$. 
However, Doering et al. defined 10 indicators to assess mercury exposure in gold miners based on a medical score and on increased mercury levels in blood, hair and urine ${ }^{[10]}$. However, no definitive standard for the diagnosis of chronic mercury intoxication has yet been established. The soluble characteristics of elemental mercury make it highly diffusible and capable of passing through cell membranes as well as the blood-brain barrier to reach target organs ${ }^{[1]}$. The central nervous system is thought to be the organ most affected by chronic exposure to elemental mercury. Vapour containing elemental mercury is absorbed easily by the lungs and then reaches the blood-brain barrier ${ }^{[11]}$. Mercury has been identified as a neurotoxin that damages the cerebellum ${ }^{[10]}$. At low levels of exposure, non-specific symptoms occur such as anorexia, weight loss, weakness, fatigue and headaches ${ }^{[9]}$. At high levels of exposure, the many clinical symptoms include muscle fasciculations, polyneuropathy with neuromuscular changes on electromyography, an abnormal Romberg test, dysdiadochokinesis and difficulty with heel-to-toe gait. Cognitive impairment has also been reported, with behavioural and personality changes, poor concentration, emotional lability, memory loss, depression, insomnia, delirium and hallucinations ${ }^{[3,9]}$. Strict avoidance of exposure may induce regression of some symptoms but with persistence of some others depending on the concentration and duration of exposure ${ }^{[3]}$. Our patient had severe neurological symptoms that completely regressed after gold mining cessation.

Elemental mercury is excreted by the kidneys where it can accumulate and cause renal damage ${ }^{[11]}$. Mercury vapour may cause mild transient proteinuria as observed in our case, but can also induce frank proteinuria, haematuria, albumin excretion and oliguria by targeting the proximal convoluted tubules ${ }^{[3]}$. Decreased renal output and renal failure have also been reported with long-term exposure ${ }^{[3,11]}$.

Cutaneous manifestations are well described with inhalation and with oral and direct dermal exposure to mercury ${ }^{[3]}$. Both systemic and topical contact may provoke allergic contact dermatitis (ACD), which is the most common skin reaction to mercury. ACD is characterized by painful or mildly pruritic eczematous eruptions ${ }^{[7]}$. Prolonged exposure to elemental mercury may cause acrodynia (formerly referred to as Pink disease), but it is restricted to infants and young children ${ }^{[11]}$. Dermal exposure can result in erythematous and peeling skin on the palms and soles ${ }^{[3]}$. Inhalation of mercury vapour can within a few days also cause acute generalized exanthematous pustulosis, an erythematous maculopapular rash with small sterile non-follicular pustules. Eruption usually begins in the proximal extremities and flexural areas and then becomes diffuse and symmetric. Significant desquamation can also be seen on the hands and feet, followed by spontaneous complete resolution 2 weeks later. Most probably, this was the type of cutaneous mercury toxicity seen in our patient, along with palmoplantar desquamation.

Gastrointestinal manifestations have been reported after ingestion of inorganic mercury which, unlike elemental mercury, is easily absorbed by the gut ${ }^{[11]}$. Inorganic mercury is corrosive to the gastrointestinal tract, causing stomatitis, excessive salivation, severe abdominal pain, diarrhoea, nausea, vomiting, ulceration and haemorrhagic lesions $\mathrm{s}^{[3,11]}$.

Low exposure to mercury has been reported to play a role in immune system dysregulation ${ }^{[12,13]}$. It may trigger autoimmune diseases such as systemic lupus erythematosus and multiple sclerosis ${ }^{[14-16]}$. An aberrant immune response resulting in inflammatory bowel disease-like lesions has been associated with mercury intoxication ${ }^{[12]}$. Heis et al. reported a case of unexplained haemorrhagic colitis after acute mercury exposure in a patient without a history of inflammatory bowel disease ${ }^{[12]}$.

The concomitant presentation in our patient of neurological, cutaneous and gastrointestinal clinical findings that completely disappeared after cessation of exposure suggests that granulomatous ileitis could be related to systemic mercury intoxication rather than Crohn's disease. Further studies are needed to confirm the potential role of mercury in granulomatous ileitis.

The management of elemental mercury poisoning is not well defined. Treatment modalities that may be helpful include specific therapy such as chelation (e.g. dimercaprol, succimer, unithiol, penicillamine) or non-specific therapeutic measures such as intravenous hydration and symptomatic treatment ${ }^{[4]}$. In our case, the patient received a systemic corticosteroid with significant clinical improvement. Whether steroids play a specific role in mercury intoxication treatment or reduce systemic inflammation requires further research and investigation.

\section{CONCLUSION}

In our patient, chronic mercury exposure induced many clinical anomalies due to the simultaneous and severe involvement of multiple organs. Symptomatic treatment and corticosteroid administration ensured rapid clinical improvement.

Chronic mercury poisoning could masquerade as an autoimmune or systemic inflammatory disease. Hence, physicians should be aware that low exposure to mercury, even from artisanal gold mining, might be harmful to health. Management can be simple without the need for aggressive or invasive therapeutic measures. Large case series are required in order to establish a clear management plan. 


\section{REFERENCES}

1. Syversen T, Kaur P. The toxicology of mercury and its compounds. J Trace Elem Med Biol 2012;26:215-226.

2. Bensefa-Colas L, Andujar P, Descatha A. Intoxication par le mercure. Rev Méd Interne 2011;32:416-424.

3. Risher JF. Elemental mercury and inorganic mercury compounds: human health aspects. Geneva: World Health Organization; 2003.

4. Caravati EM, Erdman AR, Christianson G, Nelson LS, Woolf AD, Booze LL, et al. Elemental mercury exposure: an evidence-based consensus guideline for out-of-hospital management. Clin Toxicol (Phila) 2008;46:1-21.

5. Tchounwou PB, Yedjou CG, Patlolla AK, Sutton DJ. Heavy metal toxicity and the environment. EXS 2012;101:133-164.

6. Graeme KA, Pollack CV. Heavy metal toxicity, Part I: arsenic and mercury. J Emerg Med 1998:16:45-56.

7. Dantzig PI. A new cutaneous sign of mercury poisoning? J Am Acad Dermatol 2003;49:1109-1111.

8. Lucchini R, Cortesi I, Facco P, Benedetti L, Camerino D, Carta P, et al. Neurotoxic effect of exposure to low doses of mercury. Med Lav 2002;93:202-214.

9. Bernhoft RA. Mercury toxicity and treatment: a review of the literature. J Environ Public Health 2012;2012:460508.

10. Doering S, Bose-O'Reilly S, Berger U. Essential indicators identifying chronic inorganic mercury intoxication: pooled analysis across multiple cross-sectional studies. PLoS One 2016;11:0160323

11. Boyd AS, Seger D, Vannucci S, Langley M, Abraham JL, King LE. Mercury exposure and cutaneous disease. J Am Acad Dermatol 2000;43(1 Pt 1):81-90.

12. Heise LA, Wagener BM, Vigil JR, Othman M, Shahinpoor P. Hemorrhagic colitis secondary to acute elemental mercury vapor poisoning. Am J Gastroenterol 2009;104:530-531.

13. Elli L, Bardella MT, Pigatto PD, Guzzi G. Mercury vapor overexposure and hemorrhagic colitis. Am J Gastroenterol 2009;104:2124.

14. Crowe W, Allsopp PJ, Watson GE, Magee PJ, Strain JJ, Armstrong DJ, et al. Mercury as an environmental stimulus in the development of autoimmunity - a systematic review. Autoimmun Rev 2017;16:72-80.

15. Mok CC, Leung B, Fong B, Wong CK. THU0300 serum mercury level and disease activity of systemic lupus erythematosus (SLE): a case-control study. Ann Rheum Dis 2013;72(Suppl 3):A267-A267.

16. Attar AM, Kharkhaneh A, Etemadifar M, Keyhanian K, Davoudi V, Saadatnia M. Serum mercury level and multiple sclerosis. Biol Trace Elem Res 2012;146:150-153. 